\title{
Vehicle-to-Vehicle Wireless Real Time Image Transmission for Traffic Awareness
}

\author{
D V S Ramanjaneyulu \\ Assistant Professor \\ M.Tech, \\ Vel Tech Dr.RR \& Dr.SR technical university
}

\author{
Gerardine Immaculate Mary \\ Assistant Professor (Sr.), \\ M.Tech, \\ VIT university
}

\begin{abstract}
In recent years the number of vehicles in roads have increased, which has causes high density in traffic and further problems like accident and road congestion. A solution regarding to this problem is vehicle-to-vehicle communication, where vehicles are able to communicate with their neighboring vehicles even in the absence of central base station to provide safe and more efficient road information. The goal of this paper is to communicate among vehicles for the awareness of real time traffic by sending images of the road situation at that particular instant of time. One vehicle sends information to the vehicle behind it, and so on. By using this image we find the updated traffic information. Here we are using WAVE (Wireless Access in Vehicular Environment), new wireless technology for vehicle communication, which is operated in the range of 5.850-5.925 GHz.
\end{abstract}

\section{Keywords}

IEEE 802.11p, WAVE, Matlab, Vehicle to Vehicle Communication, Dedicated Short Range Communications DSRC OFDM...

\section{INTRODUCTION}

Traffic congestion on the road is a large problem today in major cities. The congestion and related vehicle accommodation problem is accompanied by a constant threat of accidents as well. The absence of road traffic safety takes a toll of precious lives and passes a dire threat to our environment. According to National Highway Traffic Safety Administration (NHTSA) [9],

- $\quad 6.3$ million Traffic accidents were reported.

- 43,000 people were killed.

- Millions of people were injured.

- The economy effects caused due to these accidents were more than $\$ 230$ billion.

By these reasons, we need an effective communication like Vehicle to vehicle vehicular communication and networks through which vehicles and road side units communicate each other. The transferred information with this type of communication is traffic information.

Vehicular communication systems are effective in decreasing the accidents and traffic congestions. In recent years the research on vehicle to vehicle communication is increasing. IEEE 802.11p defines an international standard for wireless communication on vehicle and is known as wireless access in vehicular environment (WAVE) [3].
Vehicular communication will offer a wide range of applications such as providing traffic management which are real time data for responding to road congestions. In other hand finding the better path by accessing to the real time data is the other advantage of vehicular communication system which causes saving the time and fuel and provide large economic benefits.

\section{ROLE OF COMMUNICATION BASED VEHICULAR SYSTEM}

Cons for using sensors are limited range, limited field view and also expensive. Using wireless communication gives 360degrees driver situation and awareness.

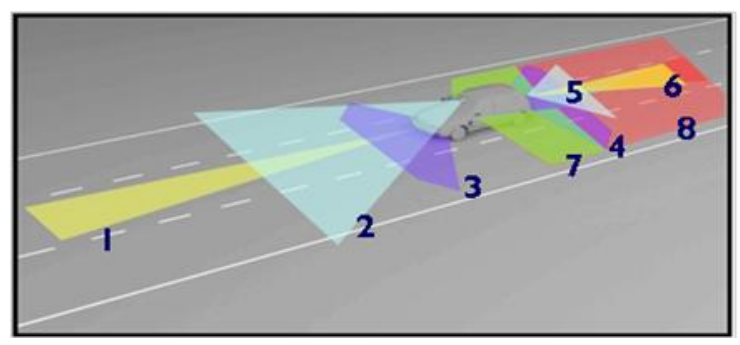

Figure1 using sensors in car

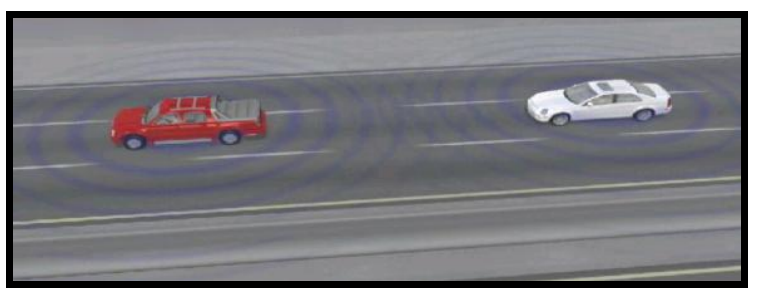

Figure2 use of wireless communication in cars

\section{OVERVIEW OF WAVE SYSTEM}

IEEE 802.11p and IEEE 1609.xx are called wireless access in vehicular environment (WAVE) and is the next generation dedicated short range communication (DSRC) technology which provides high speed vehicle to vehicle (V2V) communication. It is operated at $5.850-5.925 \mathrm{GHz}$ [4].

Total $70 \mathrm{MHz}$ band is divided in to 7 channels with each channel having $10 \mathrm{MHz}, 2$ small zones and 2 medium zones service channels are designed for extended data transfer, 2 service channels are designed for special safety critical applications and in all channels, public safety application messages has highest priority. WAVE system adopts orthogonal frequency division multiplexing (OFDM) and 
achieve data rate up to $6-27 \mathrm{Mbp} / \mathrm{s}$. In WAVE system, coverage range is up to 1000 feets. Wave systems are based on IEEE $802.11 \mathrm{p}$ protocol which is currently under development.

\section{WAVE (WIRELESS ACCESS IN VEHICULAR ENVIRONMENT) 4.1 Wave Communication Stack:}

IEEE $802.11 p$ is the combination of both MAC and Physical layer. In this, we design a physical and MAC layer such that it should be operated in the fast and rapidly varying vehicular environment. In this we are using two types of protocol stacks [2].Internet protocol version six (IPV6), Wave short message service protocol.

The reason for having two protocol stacks is to accommodate high-priority, time sense to communicate as well as more traditional and less demanding exchanges. WSMP enables the application to send short messages and directly control certain parameters of the radio resources to maximize the probability that all the implicated parties will receive the messages in time.

\subsubsection{IEEE 1609 Series (1609.XX)}

Multi-Channel Operation(IEEE 1609.4) provides enhancement to the IEEE 802.11p MAC to support multichannel operation [5].

Wave Networking Services(IEEE 1609.3) provides addressing and routing services within a wave system [6].

Wave Resource Management (IEEE 1609.1) describes an application that allows the interaction of OBU with limited computing resources and complex process, running outside the OBU in order to give the impression that the process are running in the OBUS [7].

Wave Security Services (IEEE 1609.2) covers the format of secure messages and their processing [8].

\begin{tabular}{|l|l|l|}
\hline parameters & IEEE 802.11p & IEEE 802.11a \\
\hline Bit rate Mb/s & $\begin{array}{l}3,4,5,6,9,12,18,2 \\
4,27\end{array}$ & $\begin{array}{l}6,9,12,24,36,48,5 \\
4\end{array}$ \\
\hline modulation & $\begin{array}{l}\text { Bpsk,qpsk,16- } \\
\text { qpsk,64-qpsk }\end{array}$ & $\begin{array}{l}\text { Bpsk,qpsk,16- } \\
\text { qpsk,64-qpsk }\end{array}$ \\
\hline $\begin{array}{l}\text { OFDM symbol } \\
\text { duration }\end{array}$ & $8 \mu \mathrm{s}$ & $4 \mu \mathrm{s}$ \\
\hline Guard time & $1.6 \mu \mathrm{s}$ & $0.8 \mu \mathrm{s}$ \\
\hline FFT period & $6.4 \mu \mathrm{s}$ & $3.2 \mu \mathrm{s}$ \\
\hline Preamble duration & $32 \mu \mathrm{s}$ & $16 \mu \mathrm{s}$ \\
\hline $\begin{array}{l}\text { Sub carrier } \\
\text { frequency spacing }\end{array}$ & $0.15625 \mathrm{MHz}$ & $0.3125 \mathrm{MHz}$ \\
\hline
\end{tabular}

Table 1 Comparisons view on the key parameters of IEEE 802.11p PHY and IEEE 802.11a PHY[4].

\subsection{Functional description of wave transceiver}

In the present test case, we are using camera for taking the live video on road traffic. It is fixed on the top of the vehicle. And also we use a simple Mat lab functions to take the video and get one snapshot from that video. The Mat lab code for taking the snapshot from the webcam is as follows

vid=video input('winvideo',1,'RGB24_320x240'); start(vid);

$\mathrm{b}=$ getsnapshot $($ vid $)$

imwrite(b,'traffic.bmp');

imshow(b);

The WAVE transceiver transmits the image only when the image has more than 10 to 15 vehicles and it does this process for every 5 seconds from one vehicle to another vehicle. It follows the same sequence to the last vehicle.

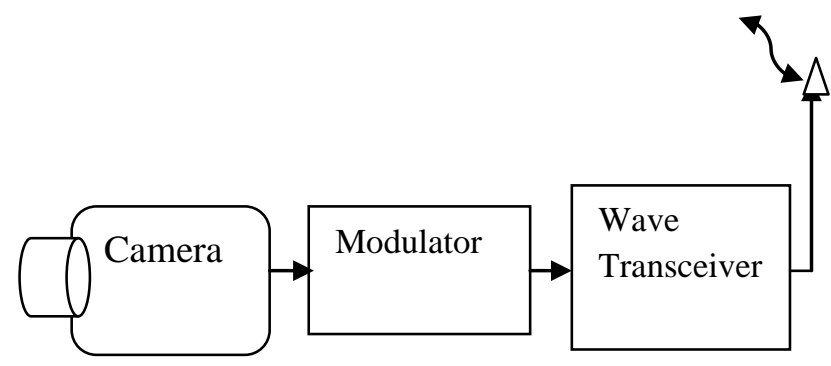

Figure 3: Functional block diagram of wave transmitter

Modulator converts the input information into IEEE $802.11 \mathrm{p}$ OFDM format. It includes different data rates which are selected according to the output of the adoptive modulation. This system uses different modulation schemes for different data rates. In this we have subsystems like padding, convolution encoder, puncturing convolution encoder, matrix interleaver, general block interleaver, Rectangular QAM because of space limitation we are not describing this subsystems.

The output of modulated video is given to the wave transceiver which can transmit the information into free space and it reaches another wave transceiver which can create its own network called wave base service sets (WBSS) and exchanges the information.

WBSS (Wave Basic Service Set)-creating scheme to support multihop data forwarding in WBSS-based IEEE 802.11(p)/1609 networks and evaluate its performances using simulations. Our simulation results show that this scheme outperforms a typical sender-centric WBSS-creating scheme on end-to-end flow goodputs under different traffic loads. 


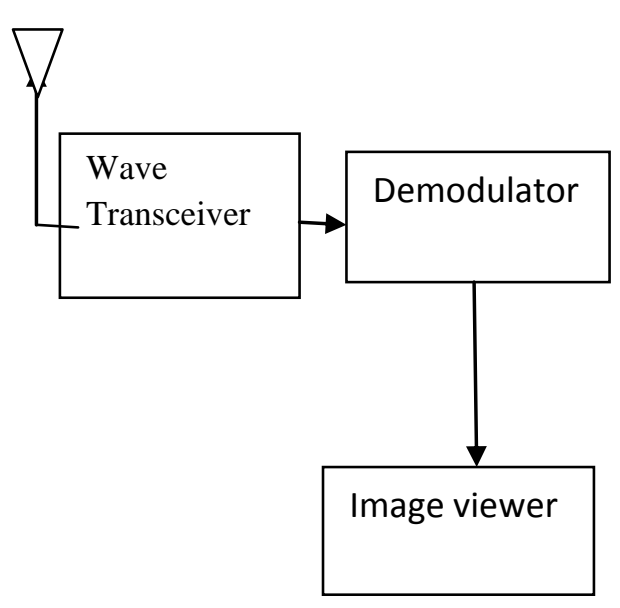

Figure 4: Functional block diagram of wave receiver

Figure. 4 shows block diagram of wave receiver in which the vehicle receives the information by using wave transceiver which is further processed and demodulated, its output is given to the image viewer, located on the OBU which shows the road condition and traffic of the road.

\subsection{Proposed Experimental Setup}

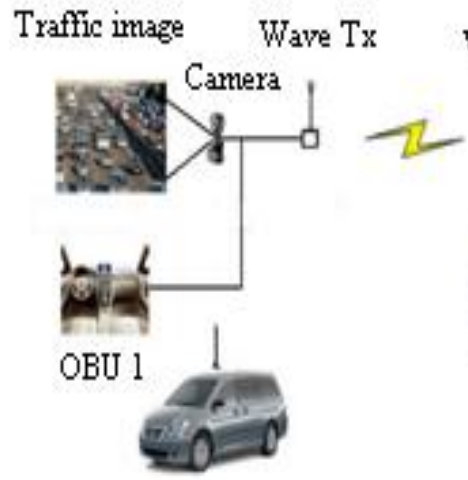

Vehicle 1

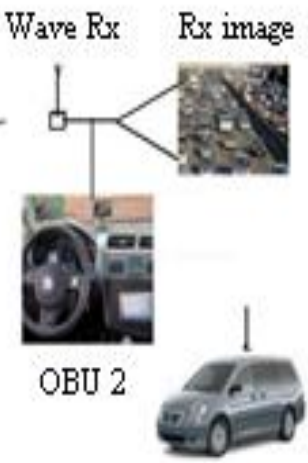

Vehicle 2
Figure 5: Experimental Setup

In the above setup, vehicle 1 captures the traffic image and transmits to vehicle 2 through WAVE transceiver.

For this experimental setup we used two computers one as a receiver and another as transmitter, the system will capture the images by using camera which is placed over the car, that image is converted by using some image processing techniques and output is given to the IEEE $802.11 \mathrm{p}$ which is physical and Mac layer of the wave system.

Figure 6 shows that graph between the number of vehicles and reception time in seconds. In the above graph, first vehicle transmission time is 2 seconds and it transmits to other vehicle in less than that time because it simply receives and transmits to the following. Finally the total 10 vehicles transmission time of 10 seconds

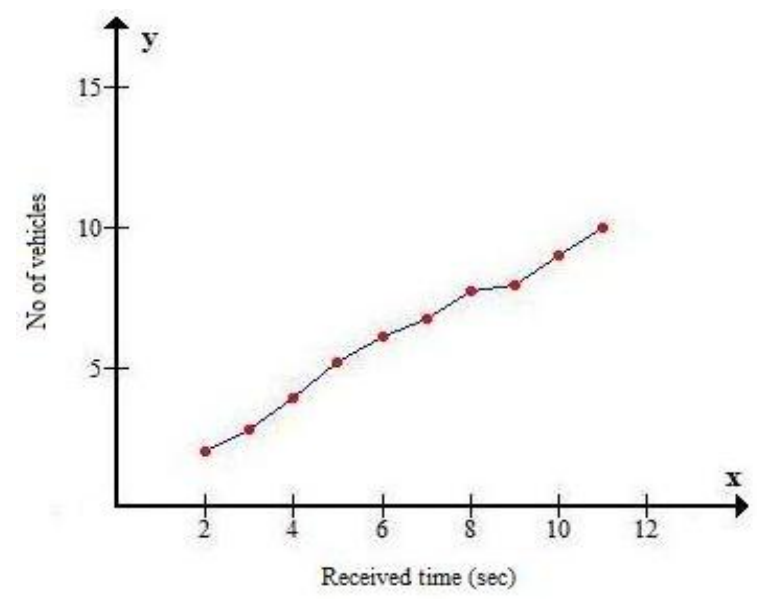

Figure 6: shows the graph between number of vehicle and it received time

\section{CONCLUSIONS}

This article presents an overview of the IEEE standards for WAVE, namely, IEEE 802.11p, IEEE 1609.1, IEEE 1609.2, IEEE1609.3, and IEEE 1609.4. Communication stack and its details, how the WBSS (wave bass service sets) are created in a network to exchange the information in the vehicles. In this we send real time traffic images to the neighboring vehicles, experimental results are communicated effectively and achieved less time delay for communicating each vehicle in the network. Future works is implementing the total protocol stack for wave and avoid the collision by sending the warning message. By using this warning message we can control the speed of the vehicle, and vehicle dynamics.

\section{REFERENCES}

[1] IEEE Std 802.11, "IEEE Standard for Information Technology-Telecommunications and Information Exchange between Systems-Local and Metropolitan Area Networks-Specific Requirements - Part 11: Wireless LAN Medium Access Control (MAC) and Physical Layer (PHY) Specifications,” 2007.

[2] IEEE Communications Magazine Roberto A. Uzcátegui, Universidad Nacional Experimental Politécnica "Antonio José de Sucre"Guillermo Acosta-Marum, Georgia Institute of Technology May 2009.

[3] Roberto A. Uzcátegui, Universidad Nacional Experimental Politécnica "Antonio José de Sucre" Guillermo AcostaMarum, Georgia Institute of Technology "WAVE: A Tutorial" TOPICSIN Automotive Networking.

[4] Arijit Khan, Shatrugna Sadhu, and Muralikrishna Yeleswarapu Dept. of Computer Science, University of California, Santa Barbara "A comparative analysis of DSRC and 802.11 over Vehicular Ad hoc Networks" May 2009.

[5] IEEE Std 1609.1-2006, Trial-Use Standard for Wireless Access in Vehicular Environments (WAVE) Resource Manager

[6] IEEE Std 1609.2-2006, Trial-Use Standard for Wireless Access in Vehicular Environments Security Services for Applications and Management Messages 
[7] IEEE Std 1609.3-2006, Trial-use Standard for Wireless Access in Vehicular Environments (WAVE) Networking Services

[8] IEEE Std 1609.4-2006, Trial-Use Standard for Wireless Access in Vehicular Environments (WAVE) MultiChannel Operation

[9] NHTSA national highway traffic safety administration.

[10] IEEE P802.11p, Draft Amendment to STANDARD FOR Information technology-Telecommunications and information exchange between systems-LAN/MAN Specific Requirements-Part 11: Wireless LAN Medium Access Control (MAC) and Physical Layer (PHY) specifications: Wireless Access in Vehicular Environments (WAVE).

\section{AUTHORS PROFILE}

D V S Ramanjaneyulu Currently working as assistant professor, school of electronics engineering in VEL Tech Dr RR \& Dr SR technical university did his diploma in Electronics \& Communication Engineering. He earned his B.Tech degree in Electronics \& Communication Engineering from NIET Guntur, in 2008. M.Tech in Automotive Electronics from VIT University. His research interests include embedded systems, communication systems, networking

Gerardine immaculate Mary pursued her M.Tech degree (wireless communication) in pondicheary engineering collage, Pondicherry. She is currently working as Assistant Professor (Sr.), School of Electronics Engineering in Vellore Institute of Technology, Vellore. She has over 12 years of experience as computer hardware engineer in the IT field. 